\title{
AVALIAÇÃO CLÍNICA DA EFICÁCIA DA COMBINAÇÃO PARACETAMOL E CAFEÍNA NO TRATAMENTO DA CEFALÉIA TIPO TENSÃO
}

\author{
GETÚLIO DARÉ RABELLO*, LUIS VICENTE FORTE**, ANTONIO CEZAR RIBEIRO GALVÃO***
}

\begin{abstract}
RESUMO - A cefaléia tipo tensão em suas duas modalidades, episódica e crônica, é a forma mais comum de dor de cabeça na população. $\mathrm{O}$ tratamento é feito com medidas não farmacológicas e farmacológicas, tanto para o ataque como profilaxia das crises. Este estudo foi realizado com 5490 pacientes, que procuraram ambulatórios e consultórios médicos em várias regiões do Brasil. Cerca de $95 \%$ apresentavam cefaléia tipo tensão episódica e $5 \%$ cefaléia tipo tensão crônica. A maioria dos pacientes apresentou crises de intensidade moderada $(62,19 \%)$. Em 5419 pacientes uma crise de cefaléia tipo tensão foi tratada com $1000 \mathrm{mg}$ de paracetamol e $130 \mathrm{mg}$ de cafeína. Em 93,98\% início de melhora foi observado em até 2 horas após a ingestão da medicação. Em 77,61\% houve reversão completa da crise em até 2 horas. Avaliação da eficácia boa/excelente foi observada em 51,93\%/37,80\% dos casos quando feita pelos médicos e em 48,51\%/40,29 quando pelos pacientes. Efeitos adversos, em geral manifestações gastrointestinais, foram observados em 5,57\%. Este estudo representa uma experiência brasileira no tratamento de ataque da cefaléia tipo tensão, demonstrando eficácia e segurança no uso da combinação paracetamol-cafeína.
\end{abstract}

PALAVRAS-CHAVE: cefaléia, tratamento, paracetamol, cafeína.

\section{Clinical evaluation of the efficacy of the acetaminophen and caffeine combination in the treatment of tension headache}

ABSTRACT - Tension type headache in both its forms, episodic and chronic, is the most common type of headache experienced by the population. The headache attack or the prevention of new crises may be treated with pharmacological as well as non-pharmacological measures. This study included 5,490 patients from outpatient clinics and medical offices covering various regions of Brazil. Approximately $95 \%$ of the subjects had episodic tension type headache, while $5 \%$ had chronic tension type headache. The majority of the patients presented with crisis of moderate intensity $(62.19 \%)$. In 5,419 patients a tension type headache crisis was treated with acetaminophen $1000 \mathrm{mg}$ and caffeine $130 \mathrm{mg}$. In $93.98 \%$, onset of relief occurred within 2 hours of taking the medication. In $77.61 \%$, complete reversion of the crisis occurred within 2 hours. Good/excellent efficacy ratings were achieved in $61.93 \% / 37.80 \%$ of the cases according to the physician's assessment and in $48.51 \% / 40.29 \%$ according to the patients' assessment. Adverse events, commonly gastrointestinal manifestations, were reported by $5.57 \%$ of the patients. This is a Brazilian study of the efficacy and safety of the combined use of acetaminophen - caffeine for the treatment of tension type headache.

KEY WORDS: headache, treatment, acetaminophen, caffeine.

Cefaléia é qualquer dor referida no segmento cefálico. Trata-se de manifestação extremamente frequente na prática clínica, e alguns dados populacionais devem ser citados: - a prevalência de cefaléia durante a vida na população geral é maior que $90 \%{ }^{1}$; - em ambulatório geral de clínica

* Mestre em Neurologia pela Faculdade de Medicina da Universidade de São Paulo (FMUSP) e Responsável pelo Ambulatório de Cefaléias da Clínica Neurológica do Hospital das Clínicas (HC)-FMUSP; **Médico Neurocirurgião, Assistente do Grupo de Neurologia e Neurocirurgia da Real e Benemérita Sociedade Portuguesa de Beneficência (Grupo Prof. Dr. Walter Carlos Pereira); ***Doutor em Neurologia pela FMUSP e Médico Assistente do HC/FMUSP. Aceite: 9-novembro-1999.

Dr. Getúlio Daré Rabello - Rua Dr. Amâncio de Carvalho 479/21 - 04012-090 São Paulo SP - Brasil. Fax 1136633875. 
médica, cefaléia representa o terceiro diagnóstico mais comum (10,3\%), sendo suplantada apenas pelas infecções de vias aéreas e dispepsias ${ }^{2}$; - em ambulatório de neurologia, cefaléia representa o mais frequente motivo de encaminhamento $(28,54 \%)$, seguido da epilepsia e transtornos mentais ${ }^{2}$.

Em 1988 a International Headache Society ${ }^{3}$ estabeleceu critérios diagnósticos para as várias modalidades de cefaléia, particularmente para as cefaléias primárias, ou seja, as não causadas por distúrbios estruturais evidentes. Isto permitiu melhor caracterização clínica, levando a avanços significativos nos estudos epidemiológicos, clínicos e terapêuticos. Entre as cefaléias primárias, a cefaléia tipo tensão (CTT) e a enxaqueca são as mais importantes.

Existem duas modalidades de CTT: episódica (CTTE), em que os quadros dolorosos ocorrem durante períodos inferiores a 15 dias ao mês, e crônica (CTTC), na qual a dor ocorre em períodos acima de 15 dias mensais, durante pelo menos 6 meses. As crises dolorosas podem durar de minutos à vários dias, apresentam caráter em pressão ou aperto, tem localização bilateral, intensidade leve a moderada e não pioram com a atividade física. Sintomas associados não costumam aparecer, embora às vezes possam ocorrer isoladamente fotofobia ou fonofobia, . Habitualmente a dor muda de localização durante a mesma crise. Dor unilateral ocorre em 10-20\% dos pacientes ${ }^{4,5}$.

Estudos sobre prevalência de CTT na população apresentam resultados diversos, devido aos diferentes critérios diagnósticos utilizados, vícios de seleção da população e falha no controle de variáveis como uso abusivo de drogas e a duração da dor de cabeça. Em países ocidentais, a prevalência em um ano é de $28-63 \%$ nos homens e $34-86 \%$ nas mulheres. A CTT é mais comum nas mulheres (relação homem/mulher $=1,04$ a 1,4) e ocorre principalmente dos 20 aos 50 anos. Cerca de $18 \%$ dos pacientes com esta forma de cefaléia interrompem suas atividades habituais e $44 \%$ tem limitação funcional. As crises ocorrem com frequência média de 2,9 dias/mês e 35/dias/ano. Cerca de um terço dos portadores tem dois ou mais ataques mensais ${ }^{6}$.

Crisp e $\operatorname{col}^{7}$, nos Estados Unidos, em 1977, encontraram prevalência durante a vida de CTT de $29 \%$ nos homens e $35 \%$ nas mulheres. Abramson e cols. ${ }^{8}$, em Israel, em 1980, observaram que a prevalência de cefaléias não enxaquecosas era de $65 \%$ para os homens e $66 \%$ para as mulheres. Rasmussen e col $^{1}$, em 1991, constataram prevalência durante a vida de CTT em $78 \%$ das pessoas (63\% nos homens e $86 \%$ nas mulheres), sendo portanto a modalidade mais frequente de cefaléia. Neste estudo a prevalência durante a vida da enxaqueca foi de $16 \%$.

Rasmussen ${ }^{9}$ em 1995 publicou estudo de prevalência em amostra representativa e aleatorizada da população de 1000 homens e mulheres, idades variando de 25-64 anos e taxa de participação de $76 \%$. A prevalência em um ano da CTTE foi de $56 \%$ nos homens e $71 \%$ nas mulheres. Já na CTTC a prevalência foi de $2 \%$ nos homens e $5 \%$ nas mulheres. A prevalência, portanto, é maior nas mulheres, corroborando dados de outros estudos. A idade média para os homens com CTT episódica/crônica foi de 42,9/44,9 anos (mediana de 43/44 anos) e para mulheres 42,3/45,4 (mediana de 42/44). A idade média nos indivíduos com CTTC foi maior que nos indivíduos com CTTE, sugerindo que as formas crônicas possam se desenvolver a partir das formas episódicas ${ }^{10}$. A evolução de uma forma episódica para forma crônica geralmente se relaciona com o uso abusivo de analgésicos. Constatou-se que no ano anterior 58\% apresentaram crises de intensidade discreta ou moderada durante apenas 1-14 dias. A prevalência de CTT ocorrendo por mais de uma vez ao mês parece ser de $20 \%$ a $30 \%{ }^{1,8,11,12}$.

Iversen e $\operatorname{col}^{13}$, na Dinamarca, observaram que 59\% da população teve CTT durante um dia ou menos, mensalmente, e 3\% teve mais de 15 dias todo mês. Estes autores citaram que as crises variam de localização entre os pacientes e mesmo no mesmo paciente entre suas diferentes crises.

O conceito mais aceito em relação à etiopatogenia da CTT é que ocorreria disfunção na modulação central da dor, seja através de mecanismos bioquímicos e mecanismos ligados à própria circuitaria neuronal. Esta modulação anormal envolveria alterações em gânglios da base, sistema límbico e neurônios no tronco encefálico, ocasionando facilitação da neurotransmissão central da 
dor e sensibilização periférica dos nociceptores miofasciais. Aliada à deficiência dos mecanismos antinociceptivos centrais, levaria à eclosão do fenômeno doloroso.

A terapêutica da CTT envolve terapias farmacológicas e não farmacológicas. Dentre as técnicas não farmacológicas de tratamento temos: terapia de apoio, técnicas de relaxamento, fisioterapia, "biofeedback" e acupuntura. Alguns estudos mostraram melhores resultados com a fisioterapia quando comparada à acupuntura ${ }^{14}$.

No tratamento farmacológico podem-se usar medicações abortivas ou de ataque, e medicações profiláticas ou preventivas. As medicações de ataque são os analgésicos comuns (ácido acetilsalicílico, paracetamol, dipirona), isolados ou associados com cafeína, ansiolíticos, codeína e anti-inflamatórios não esteróides (como naproxeno, ibuprofeno e cetoprofeno, entre outros). Em alguns países existem combinações de analgésicos com barbitúricos de ação rápida (butalbital), porém o uso delas aumenta a possibilidade de drogadição. Havendo abuso de analgésicos, o potencial para o desenvolvimento de cefaléia crônica diária aumenta.

O tratamento profilático deve ser preconizado em pacientes com crises frequentes ( $>2$ crises/ semana), de longa duração (>3-4 horas) e de intensidade moderada. Dentre as medicações preconizadas, os antidepressivos tricíclicos (amitriptilina, imipramina, nortriptilina, protriptilina, doxepin) e os inibidores seletivos de recaptação da serotonina (fluoxetina) são os mais utilizados. Não existem evidências que relaxantes musculares como baclofeno, diazepam, tizanidina, dantrolene tenham ação na CTT.

Embora o uso de analgésicos comuns seja feito há muito tempo no tratamento da CTT, estudos mostrando sua eficácia são escassos. Relatamos estudo que efetuamos.

\section{MÉTODO}

\section{Desenho do estudo}

População de 5490 pacientes portadores de cefaléia foi avaliada em estudo aberto, multicêntrico, não aleatorizado, não comparativo, por 2042 médicos e medicada com combinação farmacológica de $1000 \mathrm{mg}$ de paracetamol e $130 \mathrm{mg}$ de cafeína em um ataque agudo de cefaléia classificada como CTT. O estudo durou de julho a dezembro de 1998 .

O objetivo do estudo foi a avaliação da eficácia e segurança de dose única de combinação farmacológica de $1000 \mathrm{mg}$ paracetamol e $130 \mathrm{mg}$ cafeína no alívio da dor. Foram fornecidos 2 comprimidos a serem ingeridos concomitantemente, cada um contendo $500 \mathrm{mg}$ de paracetamol e $65 \mathrm{mg}$ de cafeína

Foram definidos os seguintes critérios de inclusão: 1. Pacientes de ambos os sexos com idade acima de 18 anos; 2. Pacientes com hipertensão arterial controlada (PA Sistólica < 140mmHg E PA Diastólica < $90 \mathrm{mmHg}$ ); 3. Pacientes que não apresentavam sintomas de enxaqueca; 4. Pacientes com potencial para engravidar, mas que fizessem uso de método contraceptivo seguro (como DIU e pílula contraceptiva); 5. Pacientes que apresentaram pelo menos 10 episódios de cefaléia; 6 . Pacientes que apresentavam cefaléia com pelo menos 30 minutos de duração; 7. Pacientes que apresentavam pelo menos duas de quatro características abaixo, em suas crises de cefaléia: - dor tipo pressão/tensão, - dor de leve a moderada intensidade, - dor de localização bilateral, - dor que não piorava com atividade física. 8. Pacientes que não apresentavam fotofobia e fonofobia, ou apenas um destes presente .

Como critérios de exclusão foram definidos os seguintes parâmetros: 1. Pacientes que apresentavam doença grave concomitante (hepatopatia, nefropatia, cardiopatia, pneumonia, úlcera péptica); 2. Mulheres grávidas ou fase de amamentação; 3. Pacientes que estavam em uso concomitante das seguintes drogas (anticoagulantes, nitratos, antiinflamatórios não esteróides, tranquilizantes e outros analgésicos); 4. Pacientes alérgicos a qualquer um dos componentes da formulação; 5.Pacientes que apresentavam vômitos durante as crises; 6. Pacientes que apresentavam história de abuso de analgésicos, definidos conforme os seguintes critérios: uso de $>10 \mathrm{cp} / \mathrm{semana}$ de analgésicos comuns, em formulações isoladas ou associadas, nos últimos 3 meses; uso de > $2 \mathrm{cp} / \mathrm{semana}$ de opiáceos, formulações isoladas ou associadas, nos últimos 3 meses; uso de $>3 \mathrm{cp} / \mathrm{semana}$ de ergóticos, em formulações isoladas ou associadas, nos últimos 3 meses. 
O estudo constou de 2 visitas com intervalo de 8 semanas. Na primeira visita, os pacientes que preencheram os critérios de inclusão e não apresentaram critérios de exclusão foram cadastrados para receber a medicação para tratar uma crise de CTT. A ficha de avaliação clínica constava de 3 partes: dados pessoais, características da cefaléia, exame clínico. O médico entregou 2 comprimidos (cp.) da formulação (cada comprimido contendo paracetamol 500mg + cafeína 65mg), a serem ingeridos de forma concomitante no momento da crise. Neste momento foram explicados os possíveis efeitos adversos, porem sem preenchimento de consentimento pósinformado, devido ao fato de tratar-se de medicação de uso habitual. Até 8 semanas da primeira visita o paciente deveria retornar à consulta e, tratada uma crise com a medicação em estudo, deveria preencher a ficha de avaliação com dados à respeito da crise tratada, eficácia da medicação e efeitos adversos. A intensidade da crise foi classificada como:

Leve, cefaléia de fraca intensidade que não inibia nem interferia com as atividades diárias;

Moderada, cefaléia de média intensidade que inibia mas não incapacitava para as atividades diárias;

Intensa, cefaléia intensa que incapacitava para as atividades diárias.

\section{Medidas da eficácia}

A avaliação da eficácia da medicação foi feita pela análise de alguns índices:

1. Tempo para ocorrer início de melhora da cefaléia;

2. Tempo de duração da cefaléia até seu desaparecimento;

3. Avaliação da eficácia segundo o médico:

Pobre, nenhum controle da cefaléia

Razoável, controle razoável da cefaléia

Bom, bom controle da cefaléia

Excelente, controle completo da cefaléia

4. Avaliação da eficácia segundo o paciente:

Pobre, nenhum controle da cefaléia

Razoável, controle razoável da cefaléia

Bom, bom controle da cefaléia

Excelente, controle completo da cefaléia

Avaliação da segurança

A segurança foi avaliada pelo relato da natureza e incidência de feitos adversos. Todos os pacientes que receberam pelo menos uma dose da medicação em estudo foram inquiridos pelo médico na segunda visita quanto aos efeitos adversos.

\section{RESULTADOS}

\section{Características dos pacientes estudados}

Foram estudados 5490 pacientes , 2764 (50,35\%) do sexo feminino e $2726(49,65 \%)$ do sexo masculino. A idade média foi 38,35 ano (mediana 37 anos), com idades entre 19 e 91 anos. Estes pacientes foram selecionados a partir de consultórios e ambulatórios médicos em várias regiões do Brasil.

Tabela 1. Tempo de história da cefaléia.

\begin{tabular}{ccc}
\hline Tempo de história & \multicolumn{2}{c}{ Total da amostra } \\
\cline { 2 - 3 } & $\mathrm{N}$ & $24,01 \%$ \\
\hline$<1$ ano & 1306 & $32,32 \%$ \\
$1-5$ anos & 1758 & $28,05 \%$ \\
$5-10$ anos & 1526 & $15,63 \%$ \\
$>10$ anos & 850 & $100 \%$ \\
Total & 5440 & $0,91 \%$ \\
Sem informação & 50 & \\
Total de pacientes & 5490 & \\
\hline
\end{tabular}


Tabela 2. Número de crises em média ao mês.

\begin{tabular}{ccc}
\hline Número de crises & $\mathrm{N}$ & $\%$ \\
\hline 1 & 698 & $12,90 \%$ \\
2 a 5 & 3383 & $62,53 \%$ \\
6 a 10 & 889 & $16,43 \%$ \\
Mais de 10 & 440 & $8,13 \%$ \\
Total & 5410 & $100 \%$ \\
Sem informação & 80 & $1,46 \%$ \\
Total de pacientes & 5490 & \\
\hline Tabela 3. Número de dias com cefaléia durante o mês & $\%$ \\
\hline Dias/mês com crise & $\mathrm{N}$ & $60,50 \%$ \\
\hline 1 a 5 & 3259 & $27,07 \%$ \\
6 a 10 & 1458 & $7,39 \%$ \\
11 a 15 & 398 & $5,05 \%$ \\
$>15$ & 272 & $100,00 \%$ \\
Total & 5387 & $1,88 \%$ \\
Sem informação & 103 & \\
\hline
\end{tabular}

\section{Características da cefaléia tipo tensão na amostra escolhida e histórico das crises}

Em relação ao tempo de existência da CTT, 56,33\% tiveram história inferior a 5 anos, em 24,01\% sendo inferior a 1 ano. Em 43,68\% a história foi superior a 5 anos, em 15,63\% sendo superior a 10 anos (Tabela 1).

Dos pacientes com informações adequadas quanto ao número de crises mensais, a maior parte apresentou menos de 5 crises ao mês $(75,43 \%)$, como está na Tabela 2.

Em relação ao número de dias de cefaléia ao mês, 94,96\% dos pacientes apresentaram crises com duração inferior a 15 dias ao mês caracterizando-se como CTTE e 5,05\% mais de 15 dias ao mês, caracterizando-se como CTTC. (Tabela 3).

As crises ocorreram no despertar, durante a manhã, tarde e noite. $\mathrm{O}$ maior percentual foi durante a tarde, ocorrendo em 57,69\% dos pacientes.

Entre os 5490 para os quais foram fornecidos os $2 \mathrm{cp}$. da formulação para tratamento de uma crise de cefaléia tipo tensão, 71 não retornaram para avaliação. Entre os 5419 que retornaram com informações a respeito da crise tratada, as principais características observadas estão nas Tabelas 4 a 8 .

Dos 5419 pacientes tratados, $302(5,57 \%)$ referiram 391 efeitos adversos (Tabela 9). Sintomas gastrointestinais foram os mais relatados, ocorrendo em 94 pacientes. Outros efeitos adversos ocorreram em percentual muito pequeno: angústia, ansiedade, cefaléia, dormência de lábios, insônia, edema de face, rubor facial, etc. Não houve qualquer efeito considerado grave pelos médicos participantes do estudo. 
Tabela 4. Intensidade da crise tratada

\begin{tabular}{lcc}
\hline Intensidade da crise & $\mathrm{N}$ & $\%$ \\
\hline Leve & 1225 & $22,80 \%$ \\
Moderada & 3341 & $62,19 \%$ \\
Intensa & 806 & $15,00 \%$ \\
Total & 5372 & $100 \%$ \\
Sem informação & 47 & \\
Total de pacientes & 5419 & \\
\hline
\end{tabular}

Tabela 5. Tempo para início de melhora da cefaléia pós ingestão da medicação.

\begin{tabular}{lcc}
\hline \multirow{2}{*}{ Tempo } & \multicolumn{2}{c}{ Número de pacientes } \\
\cline { 2 - 3 } & $\mathrm{N}$ & $\%$ \\
\hline 1/2 hora & 2366 & $45,51 \%$ \\
1 hora & 1962 & $37,74 \%$ \\
2 horas & 558 & $10,73 \%$ \\
3 horas & 130 & $2,50 \%$ \\
4 horas & 50 & $0,96 \%$ \\
5 horas & 24 & $0,46 \%$ \\
horas & 25 & $0,48 \%$ \\
Não houve melhora & 84 & $1,62 \%$ \\
Total & 5199 & $100 \%$ \\
Sem informação & 220 & \\
Total de pacientes & 5419 &
\end{tabular}

Tabela 6. Tempo de duração da cefaléia pós ingestão da medicação

\begin{tabular}{lcc}
\hline \multirow{2}{*}{ Tempo } & \multicolumn{3}{c}{ Número de pacientes } \\
\cline { 2 - 3 } & $\mathrm{N}$ & $\%$ \\
\hline $1 / 2$ hora & 791 & $15,26 \%$ \\
1 hora & 1859 & $35,86 \%$ \\
2 horas & 1373 & $26,49 \%$ \\
3 horas & 506 & $9,76 \%$ \\
4 horas & 226 & $4,36 \%$ \\
5 horas & 105 & $2,03 \%$ \\
6 horas & 137 & $2,64 \%$ \\
Não cessou após 6 horas & 187 & $3,61 \%$ \\
Total & 5184 & $100 \%$ \\
Sem informação & 235 & \\
Total de pacientes & 5419 & \\
\hline
\end{tabular}


Tabela 7. Eficácia da medicação avaliada pelo médico

\begin{tabular}{lcc}
\hline & \multicolumn{2}{c}{ Número de pacientes } \\
\cline { 2 - 3 } & $\mathrm{N}$ & $\%$ \\
\hline Pobre & 104 & $1,93 \%$ \\
Razoável & 451 & $8,35 \%$ \\
Bom & 2804 & $51,93 \%$ \\
Excelente & 2041 & $37,80 \%$ \\
Total & 5400 & $100 \%$ \\
Sem informação & 19 & \\
Total de pacientes & 5419 & \\
\hline
\end{tabular}

Tabela 8. Eficácia da medicação avaliada pelo paciente

\begin{tabular}{lcc}
\hline & \multicolumn{2}{c}{ Número de pacientes } \\
\cline { 2 - 3 } & $\mathrm{N}$ & $2,52 \%$ \\
\hline Pobre & 136 & $8,68 \%$ \\
Razoável & 468 & $48,51 \%$ \\
Bom & 2615 & $40,29 \%$ \\
Excelente & 2172 & $100 \%$ \\
Total & 5391 & \\
Sem informação & 28 & \\
Total de pacientes & 5419 &
\end{tabular}

Tabela 9. Efeitos adversos relatados - 302 pacientes $(5,57 \%)$

\begin{tabular}{lcc}
\hline Sintomas gastrointestinais & 104 & $26,6 \%$ \\
Sintomas de estimulação do sistema nervoso central & 38 & $9,7 \%$ \\
Outros & 249 & $63,7 \%$ \\
Total & 391 & $100 \%$ \\
\hline
\end{tabular}

\section{DISCUSSÃO}

Problemas ocorrem quando um estudo é feito para avaliar eficácia de medicação na CTT. O "International Headache Society Committee on Clinical Trials in Tension-type Headache"15 aponta estes problemas: dificuldade na classificação da CTT como CTTE e CTTC, duração muito variável de uma crise de CTT (poucos minutos até muitos dias) e incerteza do diagnóstico. Isto facilita a ocorrência de vícios de seleção dos pacientes e erros na avaliação da eficácia, devidos à própria variabilidade do tempo de duração de uma crise de CTT. Pode ser difícil discriminar entre a melhora da dor pela medicação ou pela própria evolução da crise. Esse Comitê recomenda realização de estudos cujo desenho seja aleatorizado, controlado com placebo, duplo-cego. Este tipo de avaliação, sem dúvida nenhuma, evita alguns erros dos estudos abertos no tratamento da CTT. Efeito placebo pode chegar a $30 \%$.

Apesar disso, estudos abertos com grande casuística podem nos responder quanto à eficácia de certas medicações para controle de crises. Neste estudo, o grande número de pacientes tratados $(\mathrm{N}=5419)$ pode reforçar a validade de suas conclusões. 
Dentre as medicações utilizadas no tratamento abortivo ou de ataque da cefaléia tipo tensão, paracetamol e cafeína, os fármacos utilizados neste estudo podem ser utilizados.

O acetaminofeno ou paracetamol, derivado do para-aminofenol, é considerado excelente analgésico em casos de dor discreta a moderada, alem de não provocar efeitos gastrointestinais importantes ${ }^{16}$. Tem fraca ação anti-inflamatória em relação ao ácido acetil-salicílico. Cuidados devem ser tomados devido à suas interações medicamentosas ${ }^{17}$. Altas doses podem potencializar a ação dos anticoagulantes orais. Além disso, cuidado especial deve ser tomado no uso em pacientes desnutridos e/ou alcoólatras, nos quais o risco de hepatotoxicidade é maior. A cimetidina pode servir como antídoto nesta situação ${ }^{18}$. Outra ação importante é a potencialização dos efeitos do cloranfenicol. O uso prolongado do paracetamol pode acelerar a absorção de zidovudina ${ }^{19}$. O uso de anticonvulsivantes pode potencializar hepatotoxicidade do paracetamol $^{20}$.

A cafeína é um alcalóide natural presente em várias plantas e pertence ao grupo das metilxantinas. Como medicamento é muito utilizada em associação com alcalóides do ergot, analgésicos e alcalóides da beladona, em preparações comerciais para tratamento de crises de enxaqueca. A cafeína não apenas aumenta a ação vasoconstrictora dos ergotamínicos, como também melhora a absorção oral e retal ${ }^{21}$. Também tem atividade anti-inflamatória definida, incluindo a capacidade de potencializar os efeitos de drogas analgésicas semelhantes à aspirina ${ }^{22}$. Quando administrada isoladamente cafeína não apresenta atividade analgésica significativa, o que não ocorre quando em associação. $\mathrm{O}$ mecanismo desta ação potencializadora não está esclarecido ${ }^{23}$. A cafeína é um estimulante do sistema nervoso central (SNC), sendo necessária cautela na associação com outros estimulantes centrais, como os anfetamínicos, para não induzir efeito excessivo. O uso concomitante da cafeína com inibidor da monoamino-oxidase (IMAO) pode provocar distúrbios cardíacos e hipertensão arterial. Suspeita-se que a cafeína possa reduzir a eficácia anticonvulsivante da fenitoina, sendo recomendável reduzi-la ou suspendê-la em pacientes epilépticos que falhem em responder adequadamente à concentrações terapêuticas da droga ${ }^{24}$. A cafeína opõe-se ao efeito hipnótico e sedativo dos barbitúricos, podendo induzir ao aumento da necessidade destes, facilitando assim a dependência.

A população estudada apresentou em $87 \%$ dos casos mais de uma crise mensal e $76 \%$ dos casos tiveram história superior a 1 ano. Estes dados, segundo as orientações do International Headache Society Committee on Clinical Trials in Tension-type Headache" ${ }^{15}$, permitem maior certeza quanto ao diagnóstico de CTT. O diagnóstico de CTTE em 94,95\% desta população e de CTTC em 5,05\% é condizente com a literatura, já que a prevalência de CTTC na população é de 3-5\% em diversos estudos.

Quanto à frequência da dor, $87,10 \%$ dos pacientes tiveram mais de duas crises mensais e $39,50 \%$ dos mesmos apresentaram dores durante 6 ou mais dias ao mês.

A maior parte dos pacientes apresentou cefaléia moderada (62,19\%). O "International Headache Society Committee on Clinical Trials in Tension-type Headache" ${ }^{15}$ admite que estas são as melhores crises a serem tratadas como CTT, pois as cefaléias leves podem melhorar de forma espontânea e as intensas podem ser confundidas com enxaqueca.

Após 2 horas a percepção do início de melhora da dor ocorreu em 93,98\% dos pacientes tratados. Este fato demonstra boa absorção da medicação e adequada farmacocinética da combinação farmacológica. O número de crises de cefaléia com reversão completa em 2 horas, considerado parâmetro de eficácia importante na avaliação de uma medicação, foi de 77,61\%. Este valor é superior ao índice de efeito placebo de $30 \%$ habitualmente admitido para este tipo de cefaléia.

No tratamento de qualquer tipo de dor, um índice que reflita a opinião do médico e do paciente para com o tratamento é considerado importante para avaliar o valor de uma medicação. Os números de $51,93 \% / 37,80 \%$ para médicos envolvidos no estudo e $48,51 \% / 40,29 \%$ para os pacientes que utilizaram a medicação, com relação à eficácia boa/excelente, indica satisfação com o resultado terapêutico.

Os efeitos adversos foram observados em 5,57\% dos pacientes, sendo geralmente ligados a manifestações gastrointestinais. Alguns sintomas para o lado do SNC como angústia, ansiedade, 
insônia, embora pudessem ser devidos à cafeína da formulação, podem eventualmente estar relacionados à própria crise.

Os resultados deste estudo apontam para a eficácia e segurança do uso de formulação farmacológica contendo $1000 \mathrm{mg}$ de paracetamol e $130 \mathrm{mg}$ de cafeína para o tratamento de ataque de crise de cefaléia tipo tensão, com segurança quanto a efeitos adversos.

Agradecimentos: Aos médicos que realizaram este esrudo em vários pontos do Brasil. A Paula Goldenstein Strassmann e Sandra Regina Malagutti pela avaliação estatística, e à Divisão Médica de Bristol Myers Squibb Brasil S/A.

\section{REFERÊNCIAS}

1. Rasmussen BK, Jensen R, Schroll M, Olesen J. Epidemiology of headache in a general population: a prevalence study. J Clin Epidemiol 1991;44:1147-1157.

2. Ferri-de-Barros JE, Nitrini R. Que pacientes atende um neurologista? Alicerce de um currículo em Neurologia. Arq Neuropsiquiatr 1996;54:637-644.

3. Headache Classification Committee of the International Headache Society. Classification and diagnostic criteria for headache disorders, cranial neuralgias and facial pain. Cephalalgia 1988; 8 (Suppl 7):1-96.

4. Drummond PD. Scalp tenderness and sensitivity to pain in migraine and tension headache. Headache 1987; 27:45-50.

5. Rasmussen BK. Migraine and tension-type headache in a general population: psychosocial factors. Int J Epidemiol 1992; 21:1138-1143.

6. Silberstein SD, Lipton RB, Goadsby PJ. Epidemiology and impact of headache disorders. In Silberstein SD, Lipton RB, Goadsby PJ (eds). Headache in clinical practice. Oxford: Isis Medical Media, 1998:19-30.

7. Crisp AH, Kalucy RS, McGuinness B, Ralph PC, Harris G - Some clinical, social and psychological characteristics of migraine subjects in the general population. Postgrad Med J 1977;53:691-697.

8. Abramson JH, Hopp C, Epstein LM - Migraine and non-migrainous headaches: a community survey in Jerusalem. J Epidemiol Community Health 1980;34:188-193

9. Rasmussen BK. Epidemiology of headache. Cephalalgia 1995;15:45-68.

10. Langemark M, Olesen J, Poulsen DL, Bech P. Clinical characterization of patients with chronic tension headache. Headache 1988;28:590-596.

11. Hollnagel H, Nørrelund N. Headache among 40-year-olds in Glostrup. Ugeskr Laeger 1980;142:3071-3077.

12. Nikiforow R. Headache in a random sample of 200 persons: a clinical study of a population in Northern Finland. Cephalalgia 1981;1:99-107.

13. Iversen HK, Langemark M, Andersson PG, Hansen PE, Olesen J. Clinical characteristics of migraine and episodic tensiontype headache in relation to old and new diagnostic criteria. Headache 1990; 30:514-519.

14. Carlsson J, Fahlcrantz A, Augustinsson L-E. Muscle tenderness in tension headache treated with acupuncture or physiotherapy. Cephalalgia 1990;10:131-141.

15. International Headache Society Committee on Clinical Trials in Tension-type Headache. Guidelines for trials of drug treatments in tension-type headache. International Headache Society Member's Handbook 1998/1999:134-160.

16. Insel PA. Analgesic-antipyretics and antiinflammatory agents; drugs employed in the treatment of rheumatoid arthritis and gout. In Gilman AG, Rall TW, Nies AS, Taylor P (eds.). The pharmacological basis of therapeutics. 8Ed. New York: Pergamon Press, 1990: 638-681.

17. Nitrini R, Galvão ACR, Barbosa ER, Yacubian EMT, Rabello GD. Neurologia. In Cordás TA, Barreto OCO (eds.). Interações medicamentosas. São Paulo: Lemos Editorial. 1998:179-201.

18. Lee WM. Drug-induced hepatotoxicity. N Engl J Med 1995,333 (17):1118-1127.

19. Burger DM, Meenhorst PL, Koks CH, Beijnen JH. Pharmacokinetics of zidovudine and acetaminophen in a patient on chronic acetaminophen therapy. Ann Pharmacother 1994;28:327-330.

20. Bray GP, Harrison PM, O'Grady JG, Tredger JM, William R. Long-term anticonvulsant therapy worsens outcome in paracetamol-induced fulminant hepatic failure. Human Exper Toxicol 1992;11:265-270.

21. Beaver WT. Combination analgesics. Am J Med 1984;77(3A):38-53.

22. Rall TW. Drugs used in the treatment of asthma. In: Gilmar AG, Rall TW, Nies AS, Taylor P (eds). The pharmacological basis of therapeutics. 8Ed. New York: Pergamon Press, 1990:618-637.

23. Migliardi JR, Armellino JJ, Friedman M, Gillings DB, Beaver WT. Caffeine as an adjuvant in tension headache. Clin Pharmacol Ther 1994;56:576-586.

24. Marquis JF, Carruthers SG, Spence JD, Brownestone YS, Toogood JH. Phenytoin-theophylline interaction. N Engl J Med $1982 ; 307: 1189-1190$ 